\title{
Electrostatic Model for Infrared Intensities in a Spectroscopically Determined Molecular Mechanics Force Field
}

\author{
KIM PALMO, SAMUEL KRIMM \\ Biophysics Research Division and Department of Physics, University of Michigan, 930 N. University, \\ Ann Arbor, Michigan 48109
}

Received 31 July 1997; accepted 17 December 1997

\begin{abstract}
A new electrostatic model for the calculation of infrared intensities in molecular mechanics and molecular dynamics is presented. The model is based on atomic charges, atomic charge fluxes, and internal coordinate dipoles and their fluxes. The internal coordinate dipoles are used instead of atomic dipoles, thus simplifying the derivation of parameters. The model is designed to reproduce $a b$ initio dipole derivatives, and the parameters can be obtained by (iterative) transformations from these, or by linear least squares fitting to them. A first application to linear alkanes has been made. For these molecules, the intensities can be predicted with an average accuracy of 30-40\%. (C) 1998 John Wiley \& Sons, Inc. J Comput Chem 19: 754-768, 1998
\end{abstract}

Keywords: molecular mechanics; spectroscopically determined force field; infrared intensities; electrostatic model; alkanes

\section{Introduction}

$\mathbf{T}$ he goal of our development of a spectroscopically determined force field $(\mathrm{SDFF})^{1}$ is to provide a molecular mechanics (MM) potential energy function, in our case based on an analytical

Correspondence to: S. Krimm; e-mail: skrimm@umich.edu Contract/grant sponsors: Polymers and Molecular Biophysics Programs of the NSF; Wright Patterson Air Force Base transformation from $a b$ initio quantities, that reproduces (in addition to structures and energies) vibrational frequencies of multiple conformations of macromolecules to spectroscopic accuracy (i.e., errors in the range of $5-10 \mathrm{~cm}^{-1}$ ). We have shown that such an approach can be implemented in the case of linear ${ }^{2}$ and branched ${ }^{3}$ saturated hydrocarbons, and thus by extension to other macromolecular systems such as polypeptides.

It is highly desirable that such an SDFF also be compatible with a model that could reproduce vibrational intensities. Aside from its direct utility, 
this would enable competent spectroscopic information to be derived from Fourier inversion of molecular dynamics (MD) simulations. In this article, we present an electrostatic model for infrared (IR) intensities that is consistent with the MM approach and that significantly improves upon previous intensity models.

It should be noted that traditional methods of reproducing IR intensities, ${ }^{4}$ making use of atomic polar tensors or of electro-optical parameters, are not useful for MM functions because their selection of, for example, atomic point charges is independent of the (all-quadratic) spectroscopic force field used to describe the molecular vibrations. In the MM approach, on the other hand, the point charges are explicitly included in the energy function and are initially optimized to reproduce relative energies of different conformers. Furthermore, charge fluxes, which are also needed to reproduce IR intensities, ${ }^{4}$ must be consistent with the intermolecular force field that gives rise to significant interaction band splittings and intermolecular normal modes, such as occur in polypeptides ${ }^{5}$ and in the formic acid dimer. ${ }^{6,7}$ Finally, we are constrained to find a model that not only provides satisfactory IR intensities for one molecule, which can always be done, but whose parameters are usefully transferable to the many other conformers of that class of molecules.

Because we have chosen to base our intensity model on $a b$ initio quantities, in this case dipole derivatives, we must also be aware of the limitation on the number of parameters that can be determined. Thus, there are too many atomic dipole fluxes to be uniquely obtained from $a b$ initio dipole derivatives whereas the internal coordinate dipole fluxes that we introduce can be directly obtained by transformation from such quantities.

In the following, we develop the theory underlying our model and this transformation, and then demonstrate its implementation for one system, the $n$-alkanes. The results show that such an electrostatic model is likely to be of sufficient generality to provide good semiquantitative IR intensities of macromolecular structures.

\section{Theory}

As usual, ${ }^{4}$ the IR absorption intensity, $I_{i r}$, of a vibrating normal coordinate, $Q_{k}$, of a molecule is proportional to the square of the derivative of the molecular electric dipole moment with respect to $Q_{k}$; that is:

$$
I_{i r}=A\left(\frac{\partial \vec{\mu}}{\partial Q_{k}}\right)^{2}
$$

where $\vec{\mu}$ is the dipole moment and $A$ is a constant. Most MM packages currently use atomic point charges to account for electrostatic intra- and intermolecular interactions and possibly for molecular dipoles. However, it is well known that static atomic point charges alone are incapable of producing correct dipole derivatives. ${ }^{4}$ As a result, in addition to static atomic charges, atomic charge fluxes have been introduced. ${ }^{4}$ Charge flux means that charge actually flows from one atom to another. As was mentioned earlier, charge fluxes can sometimes be determined from force constant data, or by fitting to band splittings and intermolecular normal modes. However, there may also be cases where the charge distributions around the atoms are deformed locally, without any net flow of charge along the bonds. This can be described in terms of atomic dipoles and atomic dipole fluxes. If no independent data are available, it may be difficult to differentiate between charge fluxes and atomic dipole fluxes in regard to their effects on the dipole derivatives. In the construction of an electrostatic model aimed at reproducing IR intensities, therefore, it seems reasonable to adopt an approach whereby charge fluxes are used only when there are clear indications that local charge redistributions are inadequate or specific spectroscopic effects require them. As we shall see, the local redistributions of charge are more convenient to describe in terms of internal coordinate dipole fluxes than in terms of atomic dipole fluxes.

We can understand the development of our model by noting that, if only atomic point charges are used, the dipole moment of a neutral molecule is:

$$
\vec{\mu}=\sum_{i} q_{i} \vec{r}_{i}
$$

where $q_{i}$ is the charge of atom $i$ and $\vec{r}_{i}$ is the radius vector of atom $i$ relative to some arbitrary origin. In molecular mechanics it is convenient, as a simple way to ensure that the molecule stays neutral, to express the charges in terms of incremental bond dipoles, such that every bond $b$ is associated with an intrinsic dipole moment:

$$
\vec{\mu}_{b}=q_{b} \vec{R}_{b}
$$


where $q_{b}$ is the incremental charge on one atom of the bond, $-q_{b}$ being the incremental charge on the other atom, and $\vec{R}_{b}$ is a vector from $-q_{b}$ to $+q_{b}$. The molecular dipole moment is then the sum of all the $\vec{\mu}_{b}$ values, and the net charge of an atom is the sum of all the incremental charges on that atom. If the charges are not constant, but depend on the deformation of the internal coordinates, we can write:

$$
q_{b}=q_{b, \text { min }}+\sum_{j} a_{b j}\left(S_{j}-S_{j, \text { min }}\right)
$$

where $S_{j}$ is an internal coordinate, $q_{b, \min }$ is the value of $q_{b}$ at the energy minimum $\vec{S}_{\text {min }}$, and:

$$
a_{b j}=\frac{\partial q_{b}}{\partial S_{j}}
$$

is the charge flux along bond $b$ due to deformation of $S_{j}$. The molecular dipole moment is then

$$
\begin{aligned}
\vec{\mu} & =\sum_{b} q_{b} \vec{R}_{b} \\
& =\sum_{b}\left[q_{b, \text { min }}+\sum_{j} a_{b j}\left(S_{j}-S_{j, \text { min }}\right)\right] \vec{R}_{b}
\end{aligned}
$$

and the dipole derivatives at $\vec{S}_{\text {min }}$ in Cartesian coordinates are:

$$
\begin{aligned}
\frac{\partial \vec{\mu}}{\partial x_{k \alpha}}= & \sum_{b} q_{b, \min } \frac{\partial \vec{R}_{b}}{\partial x_{k \alpha}} \\
& +\sum_{b}\left(\sum_{j} a_{b j} \frac{\partial S_{j}}{\partial x_{k \alpha}}\right) \vec{R}_{b}
\end{aligned}
$$

where $k$ runs over the atoms and $\alpha=1,2,3$. This can be transformed to normal coordinates using the relation:

$$
\frac{\partial \vec{\mu}}{\partial Q_{n}}=\sum_{k \alpha} \frac{\partial \vec{\mu}}{\partial x_{k \alpha}} \frac{\partial x_{k \alpha}}{\partial Q_{n}}
$$

The charge and charge flux parameters in eq. (6) can be determined via eqs. (1) and (7) by fitting to observed IR absorption intensities. This method has been used by Machida and coworkers to compute parameters for alkanes, ${ }^{8}$ aliphatic ethers, ${ }^{9}$ and amides, ${ }^{10,11}$ and also by Lii and Allinger in deriving some charge flux parameters for MM3. ${ }^{12}$

We believe, however, that a more fundamental approach, as well as better accuracy and transferability, is achieved if all parameters are determined from $a b$ initio calculations. We have, there- fore, developed an electrostatic model designed to reproduce $a b$ initio dipole derivatives. This is done in the context of an SDFF, ${ }^{1}$ which is designed to provide accurate frequencies and therefore should produce more accurate eigenvectors. This is an important requirement if we are to obtain meaningful model parameters. The force field used in this work, SDFF97, ${ }^{3}$ is an upgraded and extended version of our original SDFF for linear alkanes. ${ }^{2}$

In a first attempt to implement this approach, we optimized charge and charge flux parameters to $a b$ initio $\mathrm{HF} / 6-31 \mathrm{G}$ dipole derivatives of $n$ pentane. As expected, the resulting parameters reproduce the IR intensities satisfactorily. Using these parameters, though, we were unable to reproduce the relative energies and torsion barriers of $n$ pentane and $n$-hexane, ${ }^{2}$ because the static charges, $q_{b, \text { min }}$, came out too small compared with those required by our energy function. ${ }^{2,3}$ To yield correct energies, the charges on the hydrogens in our successful SDFF ${ }^{2,3}$ should be close to $0.1 \mathrm{e}$, whereas the above fit to the dipole derivatives gave $0.02 \mathrm{e}$ or less. (It is of interest that the value of $0.1 \mathrm{e}$ is close to the "effective charge," calculated from an experimental dipole derivative matrix, found for $\mathrm{H}$ atoms in $\mathrm{CH}_{4}, \mathrm{CH}_{3} \mathrm{CH}_{3}$, and $\mathrm{CH}_{3} \mathrm{CH}_{2} \mathrm{CH}_{3},{ }^{13}$ and close to Mulliken charges that we find in MP2/6$31++\mathrm{G}^{* *}$ calculations.) On the other hand, fixing these charges to the larger values resulted in poor IR intensities. Because some of the relative energies and barriers in $n$-pentane and $n$-hexane are almost entirely due to electrostatic interactions (in the context of our torsions and van der Waals interactions ${ }^{2,3}$ ), we therefore concluded that the charge-charge flux model was not adequate to account for IR intensities, and that at least atomic dipoles and atomic dipole fluxes also had to be added in some form to the model.

A general linear expression for an atomic dipole moment $\vec{m}_{i}$ is:

$$
\vec{m}_{i}=\vec{m}_{i, \min }+\sum_{j} c_{i j}\left(S_{j}-S_{j, \min }\right) \hat{e}_{i j}
$$

where $\vec{m}_{i, \min }$ is the atomic dipole moment at $\vec{S}_{\text {min }}$ and $c_{i j}$ are atomic dipole flux parameters that together with the unit vectors, $\hat{e}_{i j}$, determine how the atomic dipole moment changes upon deformation of the internal coordinates. Unfortunately, eq. (8) is impractical to use directly because it contains too many new parameters (three parameters per atom for the first term and three parameters per atom per internal coordinate for the second term). We also tested the approximation that $\vec{m}_{i, \min }$ and 
$\hat{e}_{i j}$ only have components along the bonds of atom $i$, but found this to be too crude. However, in order to reduce the number of new parameters and avoid the inevitably strong correlations between the parameters of eq. (5) and virtually any meaningful approximation of eq. (8), it is possible to replace the atomic dipoles and dipole fluxes with internal coordinate dipoles and dipole fluxes. For any neutral molecule, the molecular dipole moment $\vec{\mu}$ in the neighborhood of a local energy minimum $\vec{S}_{\text {min }}$ can then be described by a set of bond increment charges $q_{b}$ and a set of internal coordinate dipoles $\vec{\mu}_{j}$, such that:

$$
\vec{\mu}=\sum_{b} q_{b} \vec{R}_{b}+\sum_{j} \vec{\mu}_{j}
$$

In the linear approximation, $\vec{\mu}_{j}$ depends on the internal coordinate $S_{j}$ according to:

$$
\vec{\mu}_{j}=\vec{\mu}_{j, \text { min }}+\vec{d}_{j}\left(S_{j}-S_{j, \text { min }}\right)
$$

where the vector:

$$
\overrightarrow{d_{j}}=\frac{\partial \vec{\mu}_{j}}{\partial S_{j}}
$$

is the internal coordinate dipole flux associated with $S_{j}$. Taking into account that the charges $q_{b}$ are not constants but depend on the internal coordinates as given by eq. (4), the dipole derivatives at the energy minimum can be written:

$$
\begin{aligned}
\frac{\partial \vec{\mu}}{\partial x_{k \alpha}}= & \sum_{b} q_{b, \min } \frac{\partial \vec{R}_{b}}{\partial x_{k \alpha}} \\
& +\sum_{b, j} a_{b j} \frac{\partial S_{j}}{\partial x_{k \alpha}} \vec{R}_{b} \\
& +\sum_{j}\left(\frac{\partial \vec{\mu}_{j, m i n}}{\partial x_{k \alpha}}+\vec{d}_{j} \frac{\partial S_{j}}{\partial x_{k \alpha}}\right)
\end{aligned}
$$

(Note that even though $\vec{\mu}_{i, \text { min }}$ is a static internal coordinate dipole moment, its direction depends on the Cartesian coordinates.) Eq. (11) can be interpreted as a transformation of the derivatives of a dipole moment from internal to Cartesian coordinates. To see this we write the equation in the following form:

$$
\begin{gathered}
\frac{\partial}{\partial x_{k \alpha}}\left[\vec{\mu}-\sum_{b} q_{b, \min } \vec{R}_{b}-\sum_{b, j} a_{b j}\left(S_{j}-S_{j, \text { min }}\right) \vec{R}_{b}\right. \\
\left.-\sum_{j} \vec{\mu}_{j, \text { min }}\right]=\sum_{j} \vec{d}_{j} \frac{\partial S_{j}}{\partial x_{k \alpha}}
\end{gathered}
$$

The expression inside the brackets on the left-hand side:

$$
\begin{aligned}
\vec{M}_{s}= & \vec{\mu}-\sum_{b} q_{b, \text { min }} \vec{R}_{b}-\sum_{b, j} a_{b j}\left(S_{j}-S_{j, \text { min }}\right) \vec{R}_{b} \\
& -\sum_{j} \vec{\mu}_{j, \text { min }}
\end{aligned}
$$

is the remaining dipole moment when the effects of static charges, charge fluxes, and static internal coordinate dipoles are subtracted from the total molecular dipole moment. Thus, in our current model, $\vec{M}_{s}$ must be:

$$
\vec{M}_{s}=\sum_{j} \vec{d}_{j}\left(S_{j}-S_{j, \min }\right)
$$

Obviously:

$$
\overrightarrow{d_{j}}=\frac{\partial \vec{M}_{s}}{\partial S_{j}}
$$

so that eq. (12) can be written

$$
\frac{\partial \vec{M}_{s}}{\partial x_{k \alpha}}=\sum_{j} \frac{\partial \vec{M}_{s}}{\partial S_{j}} \frac{\partial S_{j}}{\partial x_{k \alpha}}
$$

This is the transformation of the derivatives of $\vec{M}_{s}$ from internal to Cartesian coordinates. If we invert the transformation (possible, because, with the Eckart conditions, the matrix is square), we get:

$$
\frac{\partial \vec{M}_{s}}{\partial S_{j}}=\sum_{k \alpha} \frac{\partial \vec{M}_{s}}{\partial x_{k \alpha}} \frac{\partial x_{k \alpha}}{\partial S_{j}}
$$

or, using eqns. (13) and (14):

$$
\begin{aligned}
\vec{d}_{j}= & \sum_{k \alpha}\left(\frac{\partial \vec{\mu}}{\partial x_{k \alpha}}-\sum_{b} q_{b, \min } \frac{\partial \vec{R}_{b}}{\partial x_{k \alpha}}\right. \\
& \left.-\sum_{b, i} a_{b i} \frac{\partial S_{i}}{\partial x_{k \alpha}} \vec{R}_{b}-\sum_{i} \frac{\partial \vec{\mu}_{i, \min }}{\partial x_{k \alpha}}\right) \frac{\partial x_{k \alpha}}{\partial S_{j}}
\end{aligned}
$$

Thus, if we know the static charges, the charge fluxes, and the static internal coordinate dipoles, we can directly compute the internal coordinate dipole fluxes $\vec{d}_{j}$ from the $a b$ initio dipole derivatives. This is useful if the static charges and dipoles can be determined from energy considerations, or from $a b$ initio electrostatic potentials, and (at least the most important) charge fluxes can be determined from other effects, such as shifts in vibrational frequencies and intermolecular modes. ${ }^{6,14}$ Even doing the transformation [eq. (18)] using static charges and dipoles only, could give valu- 
able clues as to what kind of charge fluxes are needed to reproduce the dipole derivatives properly. For any molecule, values for the $\vec{d}_{j}$ can always be directly computed from an $a b$ initio dipole derivative matrix. Any set of $\vec{d}_{j}, q_{b, \text { min }}, a_{b j}$, and $\vec{\mu}_{j, \min }$ compatible with eq. (18) will reproduce the $a b$ initio dipole derivatives, and hence the IR intensities, exactly. However, we also wish the parameters to be determined so as to be as transferable as possible between a set of conformers of a given class of molecules. The specific kinds of parameters optimized for such a class may well depend on which are found to be important in reproducing IR intensities, and we should be aware that this may differ between different kinds of molecules.

Finally, we note that in molecular mechanics we want to be able to compute molecular dipole moments not only at the energy minima but for any geometry. This is especially important if we want to calculate IR intensities in MD simulations. Thus, to achieve greater generality, we need to change the model slightly and replace the quantities $S_{j, \min }$ in eqs. (4) and (10) with the MM intrinsic geometry parameters $S_{j 0}$. The molecular dipole moment is then:

$$
\begin{aligned}
\vec{\mu}= & \sum_{b}\left[q_{b 0}+\sum_{j} a_{b j}\left(S_{j}-S_{j 0}\right)\right] \vec{R}_{b} \\
& +\sum_{j}\left[\vec{\mu}_{j 0}+\vec{d}_{j}\left(S_{j}-S_{j 0}\right)\right]
\end{aligned}
$$

where:

$$
q_{b 0}=q_{b, \min }+\sum_{j} a_{b j}\left(S_{j 0}-S_{j, \min }\right)
$$

and:

$$
\vec{\mu}_{j 0}=\vec{\mu}_{j, \text { min }}+\vec{d}_{j}\left(S_{j 0}-S_{j, \text { min }}\right)
$$

For the dipole derivatives we get, instead of eq. (11):

$$
\begin{aligned}
\frac{\partial \vec{\mu}}{\partial x_{k \alpha}}= & \sum_{b}\left[q_{b 0}+\sum_{j} a_{b j}\left(S_{j}-S_{j 0}\right)\right] \frac{\partial \vec{R}_{b}}{\partial x_{k \alpha}} \\
& +\sum_{b j}\left[a_{b j} \frac{\partial S_{j}}{\partial x_{k \alpha}}+\frac{\partial a_{b j}}{\partial x_{k \alpha}}\left(S_{j}-S_{j 0}\right)\right] \vec{R}_{b} \\
& +\sum_{j}\left[\frac{\partial \vec{\mu}_{j 0}}{\partial x_{k \alpha}}+\vec{d}_{j} \frac{\partial S_{j}}{\partial x_{k \alpha}}\right. \\
& \left.+\frac{\partial \vec{d}_{j}}{\partial x_{k \alpha}}\left(S_{j}-S_{j 0}\right)\right]
\end{aligned}
$$

where we have also included the possibility that the charge fluxes $a_{b j}$ may depend on conformation. For a given set of parameters, eq. (22) should be valid over a much larger range of internal coordinate deformations than eq. (11), because eq. (22) takes the deformations explicitly into account. However, eq. (22) does not lead to a direct expression for the $\vec{d}_{j}$ in the case of known charges, charge fluxes, and static internal coordinate dipoles. Thus, instead of eq. (18), we now get:

$$
\begin{aligned}
\vec{d}_{j}= & \sum_{k \alpha}\left[\frac{\partial \vec{\mu}}{\partial x_{k \alpha}}\right. \\
& -\sum_{b}\left[q_{b 0}+\sum_{i} a_{b i}\left(S_{i}-S_{i 0}\right)\right] \frac{\partial \vec{R}_{b}}{\partial x_{k \alpha}} \\
& -\sum_{b i}\left[a_{b i} \frac{\partial S_{i}}{\partial x_{k \alpha}}+\frac{\partial a_{b i}}{\partial x_{k \alpha}}\left(S_{i}-S_{i 0}\right)\right] \vec{R}_{b} \\
& \left.-\sum_{i} \frac{\partial \vec{\mu}_{i 0}}{\partial x_{k \alpha}}-\frac{\partial \vec{d}_{i}}{\partial x_{k \alpha}}\left(S_{i}-S_{i 0}\right)\right] \frac{\partial x_{k \alpha}}{\partial S_{j}}
\end{aligned}
$$

which does not yield $\vec{d}_{j}$ directly because the last term contains the derivatives of the $\vec{d}_{i}$. Therefore, in this case, the $\overrightarrow{d_{j}}$ have to be computed iteratively. This requires that a functional form of the $\vec{d}_{j}$, such as eq. (24) (next section), first be established. Alternatively, the parameters can be determined in a linear least-squares fit to the $a b$ initio dipole derivatives of a sufficiently large set of model molecules.

Even though eqs. (22) and (23) are more general, eqs. (11) and (18) are much simpler to use. Especially for nonstrained molecules, eq. (18) provides a quick method to evaluate the transferability of the $\vec{d}_{j}$, and to obtain information about the nature of charge fluxes needed to improve the transferability.

\section{Application to Linear Alkanes}

As a first application we have determined electrostatic parameters for a series of linear alkanes using $a b$ initio HF/6-31G dipole derivatives. The reason for using $\mathrm{HF} / 6-31 \mathrm{G}$ derivatives is that our SDFF for alkanes is based on structures and scaled force fields computed with this basis set. ${ }^{15}$ The following 12 molecular structures were included: ethane, propane, both conformations of butane, all four conformations of pentane, three conforma- 
tions of hexane ( $t t t, t t g$, tgg), and one conformation of heptane $(t t t)$. The ab initio dipole derivatives were calculated at energy-minimized geometries using Gaussian-94. ${ }^{16}$

Because all the molecules in the series are relatively nonstrained, we started the calculations by an evaluation of the internal coordinate dipole fluxes, $\vec{d}_{j}$, using eq. (18). For the static atomic charges we used the ones proven valid for relative energies and barriers; ${ }^{2,3}$ that is, $0.1 \mathrm{e}$ for hydrogen, and $-0.3 \mathrm{e}$ and $-0.2 \mathrm{e}$ for methyl and methylene carbons, respectively. As expected, the IR intensities produced by only these atomic charges are extremely crude and in many cases deviate from (exceed) the ab initio values by an order of magnitude. The static internal coordinate dipoles in this case were assumed to be negligible, because the energies of, and barriers between, conformers could be well accounted for by these charges. We note that this may not be true in other systems. ${ }^{6}$ The usual redundant internal coordinates were used. Thus, all six valence angles around an $s p^{3}$ carbon atom were included. The local redundancies, which have to be taken into account when using eq. (18), were treated with the method described by Pulay and Fogarasi. ${ }^{17}$ The values for the $\vec{d}_{j}$ thus obtained were then transformed into local internal coordinate systems. For each bond and angle three mutually perpendicular unit vectors were defined. For bonds, these consist of one vector along the bond, and two vectors (one arbitrary) perpendicular to the bond. For angles, the first vector bisects the angle, the second vector lies in the plane of the angle (perpendicular to the first vector), and the third vector is the vector product of the first and the second vectors. Torsion dipole flux was found to be insignificant in this class of molecules and was therefore neglected. In each case the internal coordinate dipole flux can then be written:

$$
\overrightarrow{d_{j}}=\sum_{i=1}^{3} d_{j i} \hat{e}_{j i}
$$

where $d_{j i}$ are scalar constants and $\hat{e}_{j i}$ are the internal coordinate unit vectors.

The initial calculations showed that no bonds other than $\mathrm{C}-\mathrm{H}$ bonds had significant dipole flux, and that the components perpendicular to a bond were small compared to the parallel component but still non-negligible. All angles were found to have significant dipole flux, the biggest components always being along the bisector of the angle, but with nonzero contributions also in the other directions. Internal coordinate dipole flux compo- nents that are perpendicular to the bond or the angle are inconvenient to use in molecular mechanics, because such components are bound to depend significantly on atoms other than those forming the bond or the angle. This makes it more difficult to obtain transferable parameters. We therefore decided to have the charge flux terms account for the perpendicular dipole flux components of bonds and angles. This is a somewhat arbitrary choice, but for alkanes there is no easy way to distinguish between dipole derivative contributions stemming from charge fluxes on the one hand, and from internal coordinate (or atomic) dipole fluxes on the other. (For other molecules, charge fluxes may be constrained by vibrational properties such as band splittings and intermolecular modes; in such cases, it may be necessary to include some perpendicular internal coordinate fluxes.) Test calculations on the small molecules (ethane, propane, butane) showed that the following three types of charge flux were needed: (1) nearest-neighbor bond-bond; (2) bond-angle, where the bond shares the central atom of the angle, but is not part of the angle; and (3) bond-angle, where the bond shares one of the angle's end atoms, but is not part of the angle. Charge flux terms of type (3) also turned out to be conformation-dependent. If the angle is formed by the atoms $\mathrm{ABC}$ and the bond is between atoms $\mathrm{C}$ and $\mathrm{D}$, then the charge flux parameter depends on the dihedral angle $\phi=\mathrm{ABCD}$ according to:

$$
a_{b j}=a_{b j, 0} \cos ^{2} \phi
$$

Charge flux terms beyond these types do not improve the transferability of the dipole fluxes. The following sign convention is used in the charge flux terms. For a charge increment of $\Delta q=$ $a_{b j}\left(S_{j}-S_{j 0}\right)$, the atom that the bond $\vec{R}_{b}$ shares with the internal coordinate $S_{j}$ [cf. eq. (19)] is given a charge increment of $+\Delta q$, whereas the other atom of $\vec{R}_{b}$ is given a charge increment of $-\Delta q$.

After the initial evaluation, eq. (22) was used to further optimize some of the bond and angle dipole fluxes, together with charge flux terms of the types just mentioned. The optimization was a linear least squares fit to the $a b$ initio dipole derivatives. To ensure proper weighting of the data points, both sides of eq. (22) were transformed to normal coordinates using eq. (7), and the transformed dipole derivatives were the ones actually used in the fit. Molecular mechanics eigenvectors were used, calculated at the HF/6-31G equilibrium geometries. 
TABLE I.

\section{SDFF Electrostatic Parameters for Linear}

\section{Alkanes. $^{\mathrm{a}}$}

\begin{tabular}{|c|c|c|}
\hline Bond $^{\mathrm{b}}$ & $\begin{array}{c}\text { Static } \\
\text { charge }\left(q_{b 0}\right)\end{array}$ & $\begin{array}{l}\text { Bond dipole } \\
\text { flux }\left(d_{j}\right)\end{array}$ \\
\hline \multirow[t]{2}{*}{$\mathrm{C}-\mathrm{H}$} & 0.1 & -0.3207 \\
\hline & \multicolumn{2}{|c|}{ Angle dipole flux } \\
\hline Angle $^{\mathrm{b}}$ & $\begin{array}{c}\text { Along } \\
\text { bisector }\left(d_{j 1}\right)\end{array}$ & $\begin{array}{c}\text { Perpendicular } \\
\text { to bisector }\left(d_{j 2}\right)^{\circ}\end{array}$ \\
\hline $\mathrm{C}-\mathrm{C}-\mathrm{C}$ & 0.0770 & 0.0 \\
\hline $\mathrm{C}_{3}-\mathrm{C}_{3}-\mathrm{H}$ & 0.0450 & 0.0 \\
\hline $\mathrm{C}_{2}-\mathrm{C}_{3}-\mathrm{H}$ & 0.0535 & 0.0 \\
\hline $\mathrm{C}_{3}-\mathrm{C}_{2}-\mathrm{H}$ & 0.0780 & 0.0191 \\
\hline $\mathrm{C}_{2}-\mathrm{C}_{2}-\mathrm{H}$ & 0.0657 & 0.0 \\
\hline $\mathrm{H}-\mathrm{C}_{3}-\mathrm{H}$ & 0.0550 & 0.0 \\
\hline $\mathrm{H}-\mathrm{C}_{2}-\mathrm{H}$ & 0.0640 & 0.0 \\
\hline \multicolumn{3}{|c|}{ Bond-bond charge flux $\left(a_{b j}\right)^{d}$} \\
\hline
\end{tabular}

\begin{tabular}{lr}
\hline $\mathrm{C}-\mathrm{C} / \mathrm{C}_{3}-\mathrm{C}_{2}$ & -0.0444 \\
$\mathrm{C}-\mathrm{C} / \mathrm{C}_{2}-\mathrm{C}_{2}$ & -0.0170 \\
$\mathrm{C}_{3}-\mathrm{H} / \mathrm{C}_{3}-\mathrm{H}$ & 0.0389 \\
$\mathrm{C}_{2}-\mathrm{H} / \mathrm{C}_{2}-\mathrm{H}$ & 0.0296
\end{tabular}

Bond-angle charge flux

$\begin{array}{cr}\text { Central atom shared }\left(a_{b j}\right) & \\ \mathrm{C}_{3}-\mathrm{C}_{2} / \mathrm{H}-\mathrm{C}_{3}-\mathrm{H} & 0.0086 \\ \mathrm{C}_{2}-\mathrm{H} / \mathrm{C}-\mathrm{C}-\mathrm{H} & 0.0106 \\ \mathrm{C}_{3}-\mathrm{H} / \mathrm{H}-\mathrm{C}_{3}-\mathrm{H} & 0.0222 \\ \text { End atom shared }\left(a_{b j, 0}\right)^{\mathrm{e}} & \\ \mathrm{C}-\mathrm{C} / \mathrm{C}-\mathrm{C}-\mathrm{C} & 0.0410 \\ \mathrm{C}-\mathrm{C} / \mathrm{C}-\mathrm{C}-\mathrm{H} & 0.0148 \\ \mathrm{C}_{2}-\mathrm{H} / \mathrm{C}-\mathrm{C}-\mathrm{H} & -0.0106\end{array}$

${ }^{a}$ The units are: static charge, e; bond dipole flux, e; angle

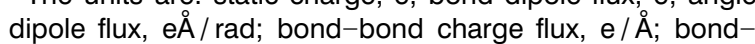
angle charge flux, e/ rad.

${ }^{\mathrm{b}} \mathrm{C}=$ methylene or methyl carbon atom; $\mathrm{C}_{2}=$ methylene carbon atom; $\mathrm{C}_{3}=$ methyl carbon atom.

${ }^{\mathrm{C}}$ If the angle is given as $\mathrm{A}-\mathrm{B}-\mathrm{C}$, the positive direction points away from atom $\mathrm{C}$.

${ }^{d}$ The first mentioned bond dipole changes when the second bond is deformed.

${ }^{e}$ Depends on conformation as given by eq. (25).

Test calculations made with eigenvectors corresponding to the full (scaled) ab initio force field ${ }^{15}$ showed that the optimization process is not very sensitive to the eigenvectors (although the intensities themselves are). The final electrostatic parameters are given in Table I. It should be noted that this set of parameters is not unique. The goal was to make it as simple as possible, but still compatible with $a b$ initio dipole derivatives and relative energies and barriers.
For some of the molecules, $a b$ initio and MM vibrational frequencies and IR intensities are given in Table II. The MM frequencies and intensities (labeled "SDFF") should be compared with those produced by the $a b$ initio dipole derivatives and scaled force fields (labeled "ab initio"). The SDFF columns are stand-alone MM results, calculated at the SDFF energy-minimized geometries. At this stage, however, we have not included the charge fluxes and internal coordinate dipole fluxes in the energy function. (In the alkanes, such inclusions have minimal effect on the charges and the energies, and only a modest effect on the intrinsic force constants. In any case, because the second derivatives of the potential energy are required to reproduce the scaled $a b$ initio force constants, ${ }^{1}$ the eigenvectors would be essentially unchanged. However, in peptides and other polar molecules these terms may give rise to much larger energy contributions and must therefore be included in the energy function.) Because the intensities are very sensitive, not only to the electrostatic model but also to the vibrational normal modes, we have included, in Table II, the column labeled " $a b$ initio/SDFF," which gives the intensities produced by the SDFF electrostatic model when the eigenvectors from the scaled $a b$ initio force fields are used. Thus, by comparing the " $a b$ initio" and " $a b$ initio/SDFF" columns, one can assess the quality of the electrostatic model under the assumption that the $a b$ initio normal modes are reproduced perfectly.

There are, indeed, some large differences between the $a b$ initio and SDFF intensities that are caused by changes in the eigenvectors rather than by failure of the electrostatic model. Examples of this are the $1333 \mathrm{~cm}^{-1}$ band in propane and the $1296 \mathrm{~cm}^{-1}$ band in $t$-butane. For these bands the electrostatic model predicts the intensities to within $20 \%$ of the $a b$ initio values when used with the $a b$ initio eigenvectors, but is off by more than a factor of 2 when the SDFF eigenvectors are used. This shows the critical importance of good eigenvectors when calculating vibrational intensities. In cases where several similar normal modes have approximately the same frequency, the composition of the eigenvectors is extremely sensitive to small changes in geometry and/or force field. This is clearly seen in the $\mathrm{C}-\mathrm{H}$ stretching region for the molecules larger than butane. The SDFF eigenvectors here are significantly different from the $a b$ initio eigenvectors. In the SDFF intensity column in Table II we therefore show intensities integrated 
ELECTROSTATIC MODEL

TABLE II.

$A b$ Initio and SDFF Vibrational Frequencies and IR Intensities.

\begin{tabular}{|c|c|c|c|c|}
\hline \multicolumn{2}{|c|}{ Frequency $\left(\mathrm{cm}^{-1}\right)$} & \multicolumn{2}{|c|}{ Infrared intensity $\left(10^{2} \mathrm{~m} / \mathrm{mol}\right)$} & \multirow[b]{2}{*}{ SDFF $^{b}$} \\
\hline$\overline{A b \text { initio }^{a}}$ & SDFF $^{\mathrm{b}}$ & $A b$ initio $^{c}$ & $A b$ initio / SDFF $^{d}$ & \\
\hline \multicolumn{5}{|l|}{ Ethane } \\
\hline 312 & 283 & 0 & 0 & 0 \\
\hline 808 & 811 & 103 & 91 & 83 \\
\hline $991^{*}$ & 996 & 0 & 0 & 0 \\
\hline $1192^{*}$ & 1199 & 0 & 0 & 0 \\
\hline 1379 & 1406 & 46 & 40 & 40 \\
\hline $1380 *$ & 1368 & 0 & 0 & 0 \\
\hline $1464^{*}$ & 1457 & 0 & 0 & 0 \\
\hline 1467 & 1446 & 213 & 200 & 203 \\
\hline 2901 & 2883 & 720 & 713 & 710 \\
\hline 2908* & 2890 & 0 & 0 & 0 \\
\hline $2952^{*}$ & 2944 & 0 & 0 & 0 \\
\hline 2976 & 2949 & 1928 & 1668 & 1673 \\
\hline \multicolumn{5}{|l|}{ Propane } \\
\hline $227^{\star}$ & 217 & 0 & 0 & 0 \\
\hline 282 & 277 & 0 & 0 & 0 \\
\hline 370 & 360 & 1 & 2 & 2 \\
\hline 739 & 744 & 38 & 34 & 29 \\
\hline 861 & 857 & 10 & 15 & 15 \\
\hline $894 *$ & 885 & 0 & 0 & 0 \\
\hline 913 & 922 & 28 & 13 & 12 \\
\hline 1055 & 1046 & 7 & 10 & 6 \\
\hline 1159 & 1162 & 18 & 17 & 19 \\
\hline 1189 & 1186 & 2 & 4 & 4 \\
\hline $1284^{*}$ & 1283 & 0 & 0 & 0 \\
\hline 1333 & 1323 & 25 & 30 & 61 \\
\hline 1368 & 1374 & 58 & 41 & 49 \\
\hline 1384 & 1375 & 42 & 57 & 13 \\
\hline $1455^{\star}$ & 1454 & 0 & 0 & 0 \\
\hline 1457 & 1459 & 0 & 8 & 5 \\
\hline 1460 & 1455 & 29 & 20 & 22 \\
\hline 1469 & 1460 & 143 & 117 & 117 \\
\hline 1471 & 1451 & 61 & 62 & 74 \\
\hline 2868 & 2860 & 459 & 409 & 518 \\
\hline 2893 & 2893 & 183 & 163 & 155 \\
\hline 2895 & 2892 & 466 & 417 & 464 \\
\hline 2900 & 2896 & 171 & 203 & 389 \\
\hline $2952^{*}$ & 2951 & 0 & 0 & 0 \\
\hline 2960 & 2951 & 406 & 299 & 254 \\
\hline 2963 & 2953 & 1254 & 1151 & 930 \\
\hline 2963 & 2955 & 706 & 642 & 577 \\
\hline \multicolumn{5}{|l|}{$t$-Butane } \\
\hline 123 & 118 & 0 & 0 & 0 \\
\hline 231 & 231 & 0 & 0 & 0 \\
\hline 262 & 253 & 1 & 5 & 4 \\
\hline $267^{*}$ & 264 & 0 & 0 & 0 \\
\hline $425^{\star}$ & 424 & 0 & 0 & 0 \\
\hline 726 & 730 & 39 & 36 & 30 \\
\hline \multirow[t]{2}{*}{$797^{*}$} & 795 & 0 & 0 & 0 \\
\hline & & (continu & & \\
\hline
\end{tabular}


PALMO AND KRIMM

TABLE II.

(Continued).

\begin{tabular}{|c|c|c|c|c|}
\hline \multicolumn{2}{|c|}{ Frequency $\left(\mathrm{cm}^{-1}\right)$} & \multicolumn{2}{|c|}{ Infrared intensity $\left(10^{2} \mathrm{~m} / \mathrm{mol}\right)$} & \multirow[b]{2}{*}{ SDFF $^{b}$} \\
\hline$\overline{A b}$ initio $^{\mathrm{a}}$ & SDFF $^{b}$ & $A b$ initio $^{c}$ & $A b$ initio / SDFF ${ }^{d}$ & \\
\hline \multicolumn{5}{|l|}{$t$-Butane } \\
\hline $828^{\star}$ & 825 & 0 & 0 & 0 \\
\hline 945 & 935 & 12 & 9 & 8 \\
\hline 964 & 968 & 85 & 77 & 73 \\
\hline 1009 & 1008 & 1 & 0 & 1 \\
\hline $1062^{\star}$ & 1056 & 0 & 0 & 0 \\
\hline $1157^{*}$ & 1157 & 0 & 0 & 0 \\
\hline $1186^{*}$ & 1173 & 0 & 0 & 0 \\
\hline 1263 & 1265 & 1 & 1 & 1 \\
\hline 1296 & 1289 & 11 & 13 & 26 \\
\hline $1300 *$ & 1308 & 0 & 0 & 0 \\
\hline $1352^{\star}$ & 1341 & 0 & 0 & 0 \\
\hline $1376^{\star}$ & 1378 & 0 & 0 & 0 \\
\hline 1377 & 1371 & 92 & 91 & 79 \\
\hline $1453^{*}$ & 1452 & 0 & 0 & 0 \\
\hline 1456 & 1458 & 24 & 22 & 22 \\
\hline $1462^{\star}$ & 1454 & 0 & 0 & 0 \\
\hline 1463 & 1452 & 152 & 118 & 111 \\
\hline $1465^{\star}$ & 1456 & 0 & 0 & 0 \\
\hline 1471 & 1458 & 93 & 107 & 118 \\
\hline $2857^{*}$ & 2866 & 0 & 0 & 0 \\
\hline 2862 & 2863 & 816 & 782 & 944 \\
\hline $2875^{\star}$ & 2893 & 0 & 0 & 0 \\
\hline 2897 & 2892 & 768 & 697 & 618 \\
\hline 2899* & 2898 & 0 & 0 & 0 \\
\hline 2899 & 2904 & 485 & 470 & 836 \\
\hline $2956^{\star}$ & 2952 & 0 & 0 & 0 \\
\hline 2960 & 2953 & 1390 & 1324 & 966 \\
\hline $2961^{*}$ & 2952 & 0 & 0 & 0 \\
\hline 2962 & 2952 & 1090 & 942 & 836 \\
\hline \multicolumn{5}{|l|}{$g$-Butane } \\
\hline 115 & 112 & 0 & 0 & 0 \\
\hline 221 & 222 & 0 & 0 & 0 \\
\hline 265 & 265 & 1 & 1 & 1 \\
\hline 328 & 321 & 0 & 0 & 0 \\
\hline 435 & 427 & 4 & 3 & 2 \\
\hline 740 & 745 & 45 & 30 & 26 \\
\hline 781 & 780 & 18 & 19 & 17 \\
\hline 821 & 823 & 1 & 1 & 1 \\
\hline 953 & 946 & 48 & 49 & 42 \\
\hline 957 & 953 & 4 & 9 & 12 \\
\hline 977 & 990 & 3 & 1 & 1 \\
\hline 1085 & 1078 & 1 & 1 & 2 \\
\hline 1139 & 1141 & 28 & 16 & 16 \\
\hline 1177 & 1175 & 1 & 2 & 3 \\
\hline 1260 & 1254 & 3 & 1 & 1 \\
\hline 1284 & 1278 & 4 & 5 & 5 \\
\hline 1338 & 1338 & 7 & 1 & 6 \\
\hline \multirow[t]{2}{*}{1346} & 1342 & 15 & 7 & 38 \\
\hline & & (cont & & \\
\hline
\end{tabular}


TABLE II.

(Continued).

\begin{tabular}{|c|c|c|c|c|}
\hline \multicolumn{2}{|c|}{ Frequency $\left(\mathrm{cm}^{-1}\right)$} & \multicolumn{2}{|c|}{ Infrared intensity $\left(10^{2} \mathrm{~m} / \mathrm{mol}\right)$} & \multirow[b]{2}{*}{$\mathrm{SDFF}^{\mathrm{b}}$} \\
\hline$\overline{A b}$ initio $^{\mathrm{a}}$ & SDFF $^{\mathrm{b}}$ & $A b$ initio $^{c}$ & Ab initio / SDFF & \\
\hline \multicolumn{5}{|l|}{$g$-Butane } \\
\hline 1375 & 1376 & 71 & 36 & 11 \\
\hline 1379 & 1379 & 56 & 55 & 51 \\
\hline 1452 & 1458 & 0 & 14 & 0 \\
\hline 1453 & 1466 & 4 & 12 & 18 \\
\hline 1462 & 1456 & 82 & 55 & 63 \\
\hline 1466 & 1462 & 50 & 32 & 50 \\
\hline 1468 & 1452 & 133 & 117 & 68 \\
\hline 1472 & 1451 & 20 & 6 & 18 \\
\hline 2856 & 2858 & 345 & 311 & 359 \\
\hline 2861 & 2895 & 252 & 219 & 251 \\
\hline 2888 & 2864 & 655 & 567 & 617 \\
\hline 2894 & 2894 & 282 & 251 & 377 \\
\hline 2900 & 2899 & 616 & 547 & 632 \\
\hline 2903 & 2896 & 83 & 126 & 179 \\
\hline 2958 & 2952 & 2 & 1 & 36 \\
\hline 2959 & 2952 & 1144 & 1019 & 778 \\
\hline 2965 & 2957 & 703 & 587 & 570 \\
\hline 2974 & 2959 & 517 & 586 & 416 \\
\hline \multicolumn{5}{|l|}{ tt-Pentane } \\
\hline $109 *$ & 109 & 0 & 0 & 0 \\
\hline 115 & 114 & 0 & 0 & 0 \\
\hline 181 & 174 & 0 & 2 & 2 \\
\hline 245 & 247 & 0 & 0 & 0 \\
\hline $254^{\star}$ & 254 & 0 & 0 & 0 \\
\hline 396 & 398 & 0 & 0 & 0 \\
\hline 399 & 396 & 0 & 3 & 4 \\
\hline 722 & 728 & 42 & 40 & 33 \\
\hline $752^{\star}$ & 753 & 0 & 0 & 0 \\
\hline 852 & 846 & 14 & 12 & 11 \\
\hline 861 & 860 & 13 & 22 & 21 \\
\hline 916 & 919 & 37 & 19 & 20 \\
\hline $977^{*}$ & 965 & 0 & 0 & 0 \\
\hline 1024 & 1025 & 28 & 13 & 12 \\
\hline 1042 & 1040 & 4 & 4 & 3 \\
\hline 1073 & 1066 & 11 & 6 & 3 \\
\hline 1153 & 1150 & 13 & 15 & 18 \\
\hline 1185 & 1168 & 1 & 2 & 2 \\
\hline $1242^{*}$ & 1242 & 0 & 0 & 0 \\
\hline 1269 & 1263 & 6 & 6 & 9 \\
\hline 1297 & 1300 & 7 & 0 & 0 \\
\hline $1301 *$ & 1315 & 0 & 0 & 0 \\
\hline 1334 & 1323 & 2 & 2 & 10 \\
\hline 1356 & 1347 & 23 & 19 & 27 \\
\hline 1377 & 1374 & 45 & 52 & 41 \\
\hline 1378 & 1376 & 30 & 24 & 17 \\
\hline 1452 & 1450 & 0 & 3 & 0 \\
\hline 1454 & 1452 & 11 & 4 & 27 \\
\hline & & (contin & & \\
\hline
\end{tabular}


PALMO AND KRIMM

TABLE II.

(Continued).

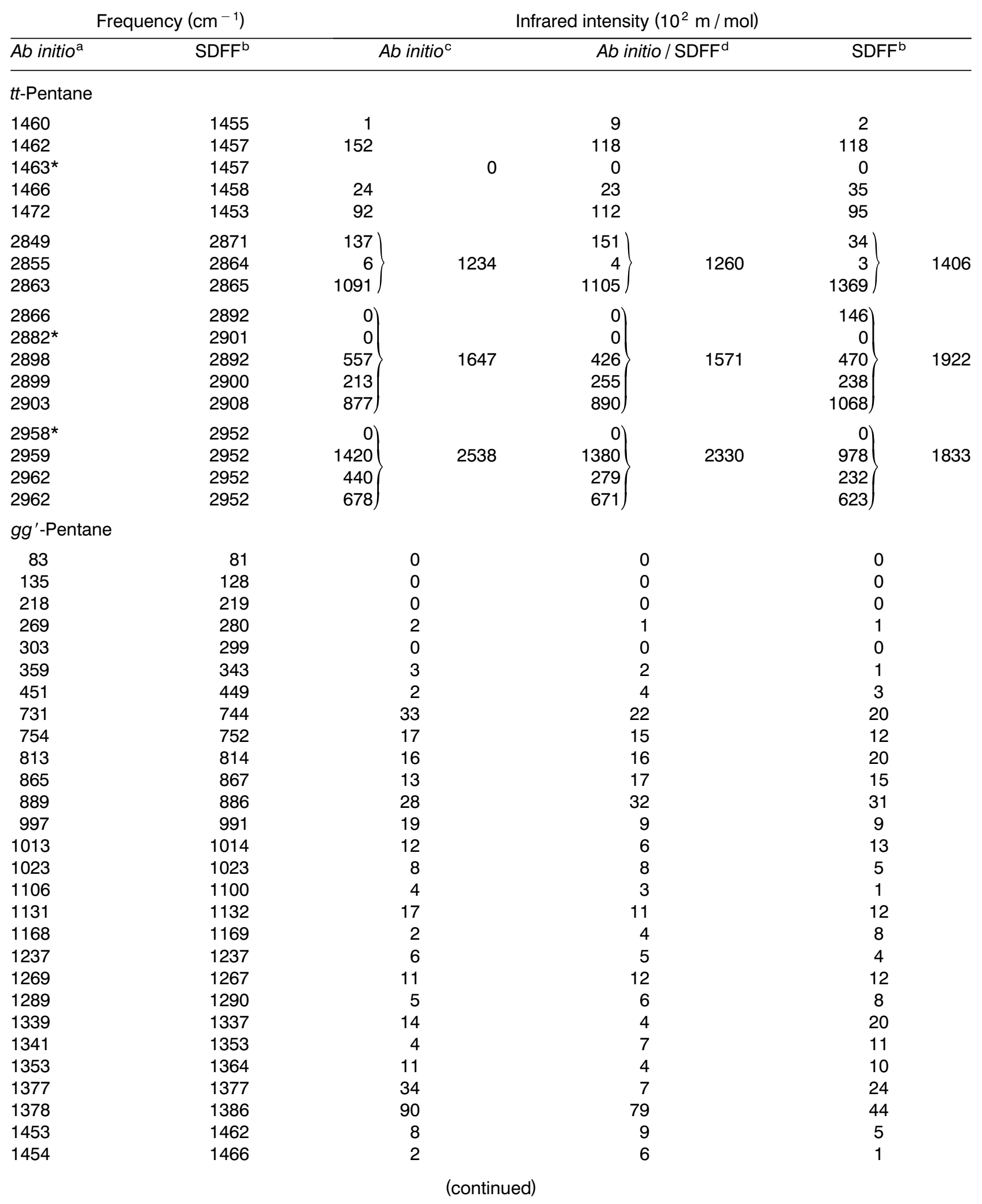


TABLE II.

(Continued).

\begin{tabular}{|c|c|c|c|c|c|c|c|}
\hline \multicolumn{2}{|c|}{ Frequency $\left(\mathrm{cm}^{-1}\right)$} & \multicolumn{6}{|c|}{ Infrared intensity $\left(10^{2} \mathrm{~m} / \mathrm{mol}\right)$} \\
\hline$\overline{A b}$ initio $^{a}$ & SDFF $^{\mathrm{b}}$ & \multicolumn{2}{|c|}{$A b$ initio $^{c}$} & \multicolumn{2}{|c|}{ Ab initio / SDFF ${ }^{d}$} & \multicolumn{2}{|c|}{ SDFF $^{\mathrm{b}}$} \\
\hline \multicolumn{8}{|c|}{$g g^{\prime}$-Pentane } \\
\hline 1458 & 1472 & 19 & & 28 & & 7 & \\
\hline 1463 & 1453 & 36 & & 21 & & 22 & \\
\hline 1466 & 1452 & 120 & & 61 & & 81 & \\
\hline 1470 & 1457 & 25 & & 32 & & 31 & \\
\hline 1474 & 1478 & 120 & & 84 & & 87 & \\
\hline 2850 & 2862 & 278 & \multirow{3}{*}{716} & 286 & \multirow{3}{*}{673} & 773 & \multirow{3}{*}{1443} \\
\hline 2857 & 2856 & 275 & & 231 & & 404 & \\
\hline 2860 & 2852 & 163 & & 156 & & 266 & \\
\hline 2882 & 2896 & 373) & \multirow{5}{*}{2531} & 256) & \multirow{5}{*}{2321} & 219) & \multirow{5}{*}{1905} \\
\hline 2892 & 2899 & 600 & & 608 & & 442 & \\
\hline 2894 & 2901 & 894 & & 834 & & $769\}$ & \\
\hline 2902 & 2893 & 260 & & 196 & & 214 & \\
\hline 2906 & 2900 & 404) & & 427) & & 261) & \\
\hline 2957 & 2950 & 422) & \multirow{4}{*}{2313} & 363) & \multirow{4}{*}{2151} & $441)$ & \multirow{4}{*}{1799} \\
\hline 2959 & 2963 & 774 & & 673 & & 474 & \\
\hline 2972 & 2952 & 743 & & 663 & & $451\}$ & \\
\hline 2986 & 2977 & 374 & & $452)$ & & 433 & \\
\hline
\end{tabular}

${ }^{a}$ Calculated at HF / 6-31G-energy-minimized geometry using scaled force field (scale factors from ref. 11). Frequencies marked with an asterisk are infrared inactive by symmetry.

${ }^{\mathrm{b}}$ Molecular mechanics calculation at SDFF-energy-minimized geometry.

${ }^{\mathrm{c}} \mathrm{HF} / \mathrm{6}-31 \mathrm{G}$ dipole derivatives and geometry. Eigenvectors calculated using scaled force field.

${ }^{\mathrm{d}}$ Eigenvectors calculated at HF / 6-31G-energy-minimized geometry using scaled force field. SDFF electrostatic parameters.

TABLE III.

Intensity Deviations ( $\mathrm{rms}$ ) in Different Ranges $\left(10^{2} \mathrm{~m} / \mathrm{mol}\right)$.

\begin{tabular}{lrrrrr}
\hline & \multicolumn{3}{c}{ Non-C-H stretching (intensity range ${ }^{\text {a }}$ ) } & \\
\cline { 2 - 4 } Molecule & $0-10$ & $10-50$ & $50-100$ & $100+$ & C-H stretching (all ranges) \\
\hline Ethane & - & 6 & - & 16 & 181 \\
Propane & 3 & 20 & 12 & 26 & 168 \\
$t$-Butane & 2 & 9 & 8 & 41 & 285 \\
g-Butane & 5 & 13 & 37 & 65 & 135 \\
tt-Pentane & 4 & 11 & 3 & 34 & 448 \\
tg-Pentane & 4 & 7 & 34 & 41 & 471 \\
gg-Pentane & 3 & 14 & 26 & 53 & 629 \\
gg'-Pentane & 3 & 8 & 46 & 36 & 486 \\
ttt-Hexane & 3 & 18 & 21 & 21 & 516 \\
ttg-Hexane & 1 & 11 & 23 & 65 & 712 \\
tgg-Hexane & 4 & 9 & 30 & 69 & 405 \\
tttt-Heptane & 3 & 15 & 12 & 21 & 30 \\
All molecules & 3 & 12 & 27 & 42 & 23 \\
rms dev\% & 66 & 39 & 37 & 21 & 48 \\
Ave dev\% & 40 & 30 & 32 & 18 & \\
No. of freqs. & 153 & 111 & 26 & 16 &
\end{tabular}

\footnotetext{
${ }^{\mathrm{a}}$ Intensities that are zero by symmetry are not included.

${ }^{\mathrm{b}}$ The $\operatorname{dev} \%$ values are defined as $100 * \operatorname{dev} / I_{\text {mid }}$, where $I_{\text {mid }}$ is the average of the highest and the lowest intensity found in the range.
} 
over the respective $\mathrm{C}-\mathrm{H}$ stretching frequency intervals. Also, in this case, as can be seen from the "ab initio/SDFF" column, the electrostatic model itself is in good agreement with $a b$ initio. The region near $1450 \mathrm{~cm}^{-1}$ is another crowded area. Here, many methyl and methylene bending modes occur with closely spaced frequencies.

The root-mean-square (rms) intensity deviations of SDFF compared with ab initio in different intensity ranges are given in Table III, separately for each molecule. With respect to the average deviations, the $a b$ initio IR intensities are reproduced with an accuracy of 30-40\%, which in many cases is sufficient for semiquantitative predictions. The electrostatic model as such is capable of significantly better predictions, but its performance is limited by the less-than-perfect SDFF eigenvectors (and probably by the restriction in the kinds and number of parameters in the model). This is seen by comparing the dev\% values in Table III with those obtained using $a b$ initio/SDFF data (defined as in Table II) instead of pure SDFF data. The corresponding $\mathrm{rms} \operatorname{dev} \%$ (ave $\operatorname{dev} \%$ ) values are 59(36), 31(24), 28(23), 14(12), and 8(7) for the $0-10$, 10-50, 50-100, $100+$, and $\mathrm{C}-\mathrm{H}$ stretching intensity ranges, respectively, which represents an average improvement of about $22 \%$ for non- $\mathrm{C}-\mathrm{H}$ stretching modes.

To give an impression of what the current SDFF predictions would look like, we have simulated a few spectra assuming Lorentzian band shapes with half-widths of $10 \mathrm{~cm}^{-1}$. Figures 1-4 show a comparison between $a b$ initio and SDFF-simulated spectra for ethane, $t$-butane, $g$-butane, and $t g g$ hexane. The spectral features are reasonably well accounted for, especially below $1300 \mathrm{~cm}^{-1}$. Of course, a condition for a simulation to be even qualitatively successful is that the positions of the bands, that is, the vibrational frequencies, are accurately reproduced. The rms frequency deviation below $1500 \mathrm{~cm}^{-1}$ for the molecules treated in this work (SDFF compared with $a b$ initio) is $6.9 \mathrm{~cm}^{-1}$.

\section{Conclusions}

We have presented a new electrostatic model, and parameters for linear alkanes, which make semiquantitative predictions of IR spectra possible in molecular mechanics and molecular dynamics calculations. Such predictions give significantly better results than previous MM models.
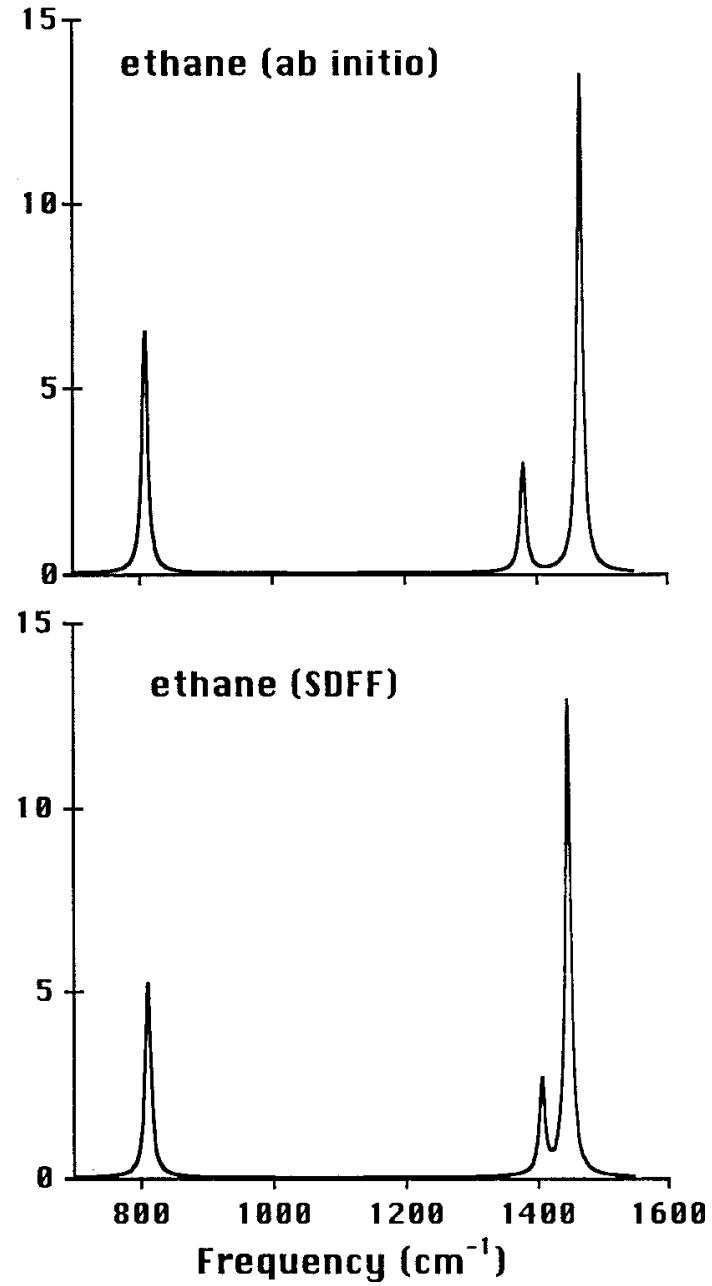

FIGURE 1. Simulated ab initio (top) and SDFF (bottom) infrared spectra of ethane. The bands consist of Lorentzian profiles with half-widths of $10 \mathrm{~cm}^{-1}$. The $a b$ initio intensities were calculated using HF / 6-31G dipole derivatives and scaled force fields. Intensities are in units of $10^{2} \mathrm{~m} / \mathrm{mol}$.

Our model of course differs from traditional (i.e., atomic polar tensor or electro-optical parameter) models in that these are based on spectroscopic force fields. Also, we have included angle dipole fluxes in addition to bond dipole fluxes to account for dipole derivatives. The parameters in our model can conveniently be obtained from $a b$ initio dipole derivatives by linear least-squares fitting. The main new quantities presented here, internal coordinate dipole fluxes, can also be determined by direct transformation (or iteratively) from $a b$ initio dipole derivatives if the other parameters are known. In planar molecules or groups, internal coordinate dipole fluxes probably provide the easi- 


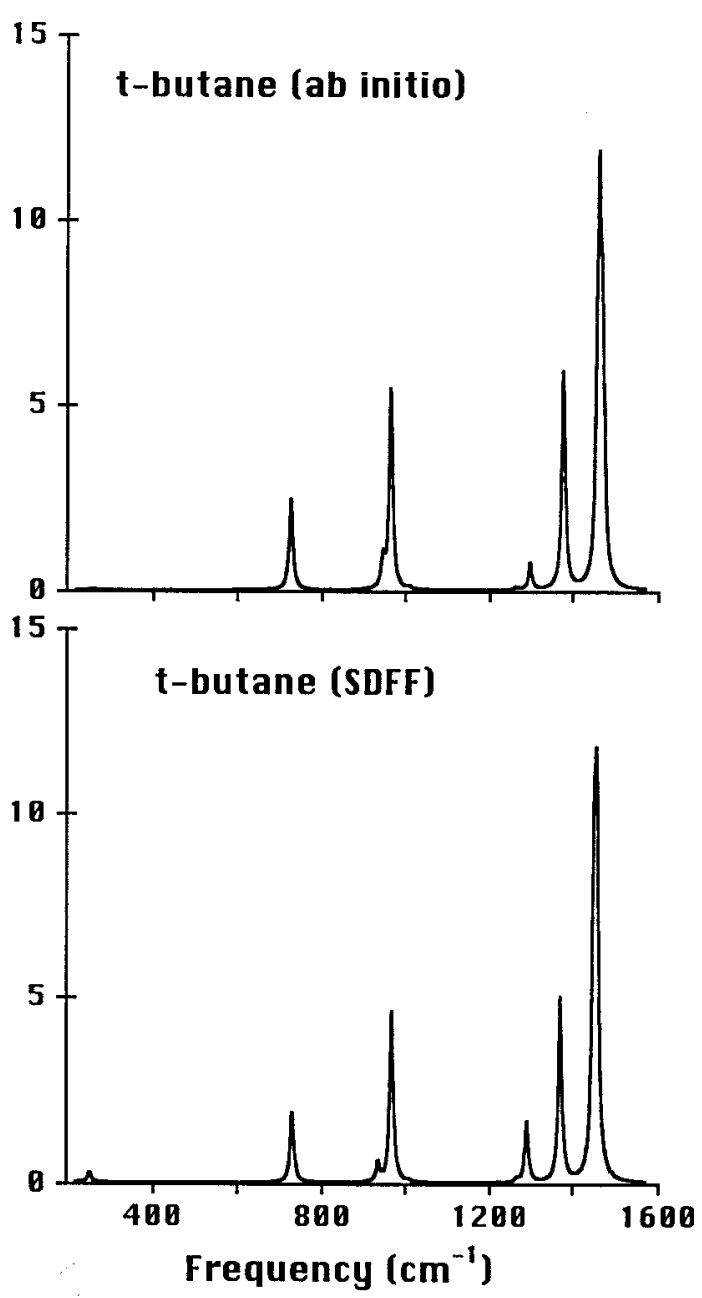

FIGURE 2. Simulated ab initio (top) and SDFF (bottom) infrared spectra of $t$-butane. Description as in Figure 1.

est way to describe out-of-plane charge redistribution, because charge fluxes do not have out-ofplane components. To be of any value, such an electrostatic model must be combined with force fields, such as SDFFs, ${ }^{2,3}$ which yield very good vibrational frequencies and eigenvectors.

The SDFF IR intensities can be improved by calculating better normal modes or by a more comprehensive electrostatic model or parameters. For example, there may be room for improvement in the conformation dependence of the parameters. Although torsion dipole fluxes in alkanes are negligible for small deformations, it is still possible that the static charges have some systematic conformation dependence that is not taken properly into account by the current set of charge fluxes. $A b$ initio investigations of electrostatic potentials of
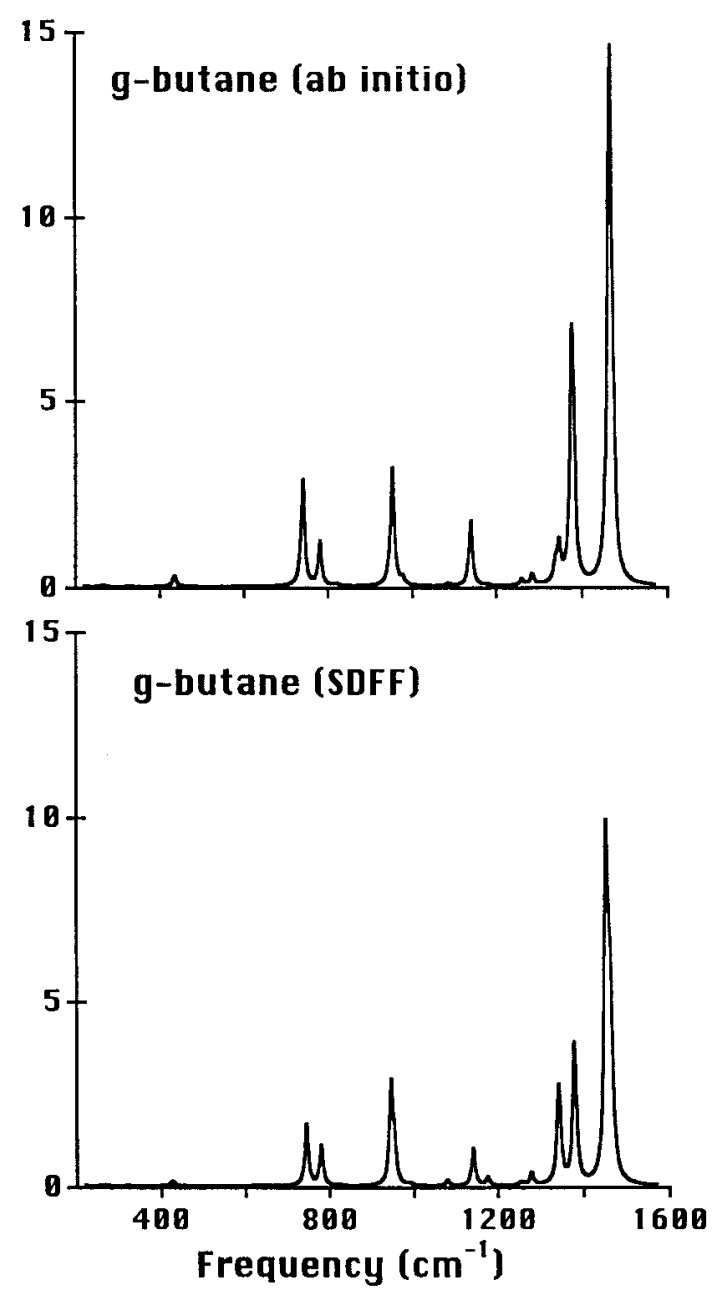

FIGURE 3. Simulated ab initio (top) and SDFF (bottom) infrared spectra of $g$-butane. Description as in Figure 1.

alkanes even seem to indicate that a net atomic charge model is not adequate and that additional charge centers are needed. ${ }^{18,19}$ However, there are also indications that the alkanes are an exception in this regard, because, although we find that atomic point charges in $N$-methylacetamide can reproduce the $a b$ initio ( $\mathrm{HF} / 6-31+\mathrm{G}^{* *}$ ) electrostatic potential (on a Merz-Singh-Kollman grid) with a relative rms error of $7 \%$, the corresponding value for $t t$-pentane is $85 \%$. Another difficulty in improving the electrostatic model for alkanes is that charge fluxes and internal coordinate (or atomic) dipole fluxes affect the dipole derivatives in the same way and cannot easily be distinguished.

A variation of the method described in this work can be used for the determination of parame- 


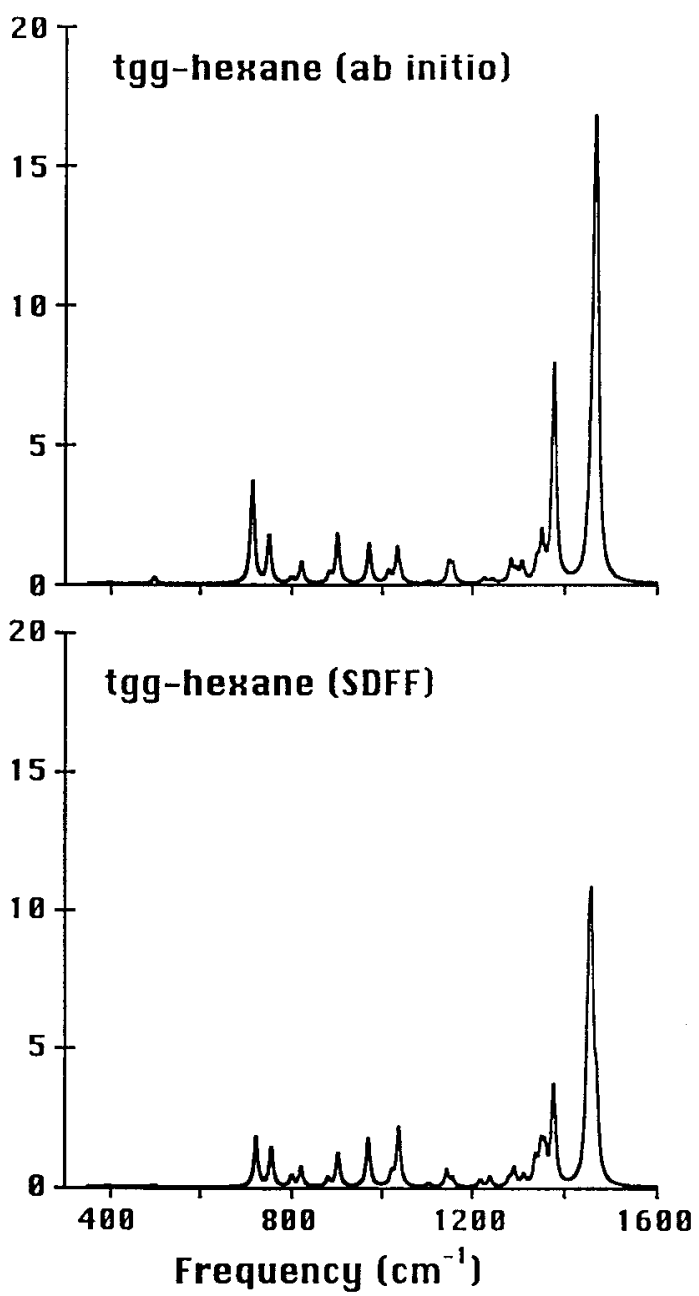

FIGURE 4. Simulated ab initio (top) and SDFF (bottom) infrared spectra of tgg-hexane. Description as in Figure 1.

ters for the calculation of conventional Raman spectra, ${ }^{20}$ preliminary results of which (including those of the present work) have been presented. ${ }^{21}$

\section{Acknowledgment}

We are grateful for the helpful discussions with Weili Qian.

\section{References}

1. K. Palmo, L-O. Pietila, and S. Krimm, J. Comput. Chem., 12, 385 (1991).

2. K. Palmo, N. G. Mirkin, L-O. Pietila, and S. Krimm, Macromolecules, 26, 6831 (1993).

3. K. Palmo, N. G. Mirkin, and S. Krimm, to be published.

4. W. Person and G. Zerbi, Eds., Vibrational Intensities in Infrared and Raman Spectroscopy, Elsevier, Amsterdam, 1982.

5. S. Krimm and J. Bandekar, Adv. Prot. Chem., 38, 181 (1986).

6. W. Qian and S. Krimm, J. Phys. Chem., 100, 14602 (1996).

7. W. Qian and S. Krimm, J. Phys. Chem., 102, 659 (1998).

8. Y. Miwa and K. Machida, J. Am. Chem. Soc., 110, 5183 (1988).

9. Y. Miwa and K. Machida, J. Am. Chem. Soc., 111, 7733 (1989).

10. E. Ganeshsrinivas, D. N. Sathyanarayana, K. Machida, and Y. Miwa, J. Mol. Struct. (Theochem), 361, 217 (1996).

11. E. Ganeshsrinivas, D. N. Sathyanarayana, K. Machida, and Y. Miwa, J. Mol. Struct., 403, 153 (1997).

12. J-H. Lii and N. L. Allinger, J. Comput. Chem., 13, 1138 (1992).

13. W. T. King, ref. 4, p. 122.

14. I. Yokoyama, Y. Miwa, and K. Machida, J. Am. Chem. Soc., 113, 6458 (1991).

15. N. G. Mirkin and S. Krimm, J. Phys. Chem., 97, 13887 (1993).

16. M. J. Frisch, G. W. Trucks, H. B. Schlegel, P. M. W. Gill, B. G. Johnson, M. A. Robb, J. R. Cheeseman, T. Keith, G. A. Petersson, J. A. Montgomery, K. Raghavachari, M. A. AlLaham, V. G. Zakrzewski, J. V. Ortiz, J. B. Foresman, J. Cioslowski, B. B. Stefanov, A. Nanayakkara, M. Challacombe, C. Y. Peng, P. Y. Ayala, W. Chen, M. W. Wong, J. L. Andres, E. S. Replogle, R. Gomperts, R. L. Martin, D. J. Fox, J. S. Binkley, D. J. Defrees, J. Baker, J. P. Stewart, M. Head-Gordon, C. Gonzalez, and J. A. Pople, Gaussian-94, Revision D.4, Gaussian Inc., Pittsburgh, PA, 1995.

17. P. Pulay and G. Fogarasi, J. Chem. Phys., 96, 2856 (1992).

18. C. Chipot, J. G. Angyan, G. G. Ferenczy, and H. A. Scheraga, J. Phys. Chem., 97, 6628 (1993).

19. D. E. Williams, J. Comput. Chem., 15, 719 (1994).

20. K. Palmo and S. Krimm, to be published.

21. K. Palmo, N. G. Mirkin, and S. Krimm, In XVth International Congress on Raman Spectroscopy, S. A. Asher and P. B. Stein, Eds., Wiley, New York, 1996, p. 208. 